\title{
ÖWAV-TAGUNGEN, ÖWAV-SEMINARE
}

Durchgängigkeit an Fließgewässern Anforderungen aus Sicht der EU-WRRL Erkenntnisse aus der Praxis

Termin: 7.-8. Mai 2009

Ort: Stadthalle, Ybbs

Leitung: DI Dr. Otto Pirker, DI Dr. Michael Hengl

Kosten (+ $20 \%$ USt.): ÖWAV-Mitglieder Euro 390,-, Nichtmitglieder Euro 250,-

\section{Alpenkonvention und Tourismus - Auditierung in Skigebieten}

Termin: 12.-13. Mai 2009

Ort: Congress, Innsbruck

Kosten (+ 20 \% USt.): ÖWAV-Mitglieder Euro 390,-, Nichtmitglieder Euro 250,-

\section{Hochwässer - Bemessung, Risikoanalyse und Vorhersage}

Termin: 26. Mai 2009

Ort: Bundesamtsgebäude, Wien

Leitung: Univ.-Prof. DI Dr. Günter Blöschl

Kosten (+ $20 \%$ USt.): ÖWAV-Mitglieder Euro 200,-, Nichtmitglieder Euro 350,-

100 Jahre ÖWAV - Fach- und Festveranstaltung

Termin: 18. Juni 2009

Ort: Galerie der Forschung, Wien

Teilnahme kostenfrei

\section{EU-Wasserrahmenrichtlinie - Nationaler Gewässerbewirtschaftungsplan (NGP)}

Termin: 22. Juni 2009

Ort: Wien

\section{UVP-Pflicht bei Golfplätzen - Vorstellung des ÖWAV-Regelblatts 216}

Termin: 24. Juni 2009

Ort: Gartenhotel Altmannsdorf, Wien

Leitung: DI Dr. Eduard Klaghofer

Kosten (+ $20 \%$ USt.): ÖWAV-Mitglieder/Mitglieder des Golfverbands Euro 200,--, Nichtmitglieder Euro 350,-

\section{Optimierte Bemessung von \\ Mischwasserentlastungsanlagen}

Termin: 30. Juni 2009

Ort: Technische Universität Graz 
Mitveranstalter: TU Graz, Institut für Siedlungswasserwirtschaft und Landschaftswasserbau, Lebensministerium

Leitung: Univ.-Prof. DDI Dr. Dr. h.c. Harald Kainz

\section{Österreichische Umweltrechtstage 2009}

Termin: 9.-10. September 2009

Ort: Universität Linz

Mitveranstalter: Institut für Umweltrecht der Universität Linz

Leitung: Univ.-Prof. Dr. Ferdinand Kerschner, o.Univ.-Prof.

Dr. Bernhard Raschauer

\section{Verpackungsverordnung neu}

Termin: 17. September 2009

Ort: Wien

\section{Speicherung, Pumpspeicherung, Betriebsführung}

Termin: 23. -24. September 2009

Ort: Graz

Leitung: Univ.-Prof. DI Dr. Gerald Zenz, Univ.-Prof. DI Dr. Peter Tschernutter

\section{3. Österreichischer Kleinkläranlagentag}

Termin: 24. September 2009

Ort: Universität für Bodenkultur Wien

Mitveranstalter: BOKU, Institut für Siedlungswasserbau, Industriewasserwirtschaft und Gewässerschutz

Leitung: Univ.-Prof. DI Dr. Raimund Haberl

\section{Internationales Anwenderforum Kleinwasserkraftwerke}

Termin: 24. -25 . September 2009

Ort: TIS Bozen (I)

Veranstalter: OTTI (ÖWAV Mitveranstalter)

Auskunft: OTTI, Fr. Leonore Nanko, leonore.nanko@otti.de, www.otti.de

Fettabscheideranlagen - Vorstellung ÖWAVRegelblatt 39 - Bemessung, Kennzeichnung, Betrieb

Termin: 6. Oktober 2009

Ort: Innsbruck

Weitere Informationen und Anmeldung:

Gesellschaft für Wasser- und Abfallwirtschaft GmbH des ÖWAV 1010 Wien, Marc-Aurel-Straße 5 , Martin Waschak, Tel. 01/535 57 20-75, waschak@oewav.at oder www oewav. at $\gg$ Veranstaltungen

Aufgrund der Voranmeldung erhalten Sie das detaillierte Veranstaltungsprogramm.
ÖWAV-KURSE

ABWASSER

24. Grundkurs für das Betriebspersonal von Kanalisationsanlagen

Termin: 4.-8. Mai 2009

Ort: Wien

Kosten (+ 20 \% USt.): ÖWAV-Mitglieder Euro 560,-, Nichtmitglieder Euro 710,-

8. ÖWAV-Kleinkläranlagenkurs für bepflanzte Bodenfilter ( $\leq 50 \mathrm{EW}$ ) (Pflanzenkläranlagen)

Termin: 8. -9. Mai 2009

Ort: Oberndorf/Melk (NÖ)

Kosten (+ 20 \% USt.): Euro 220,-

\section{Fortbildungskurs für das Betriebspersonal von Kanalisationsanlagen}

Termin: 11. -15. Mai 2009

Ort: Steyr

Kosten (+ $20 \%$ USt.): ÖWAV-Mitglieder Euro 650,--, Nichtmitglieder Euro 800,-

\section{Kanalfacharbeiterprüfung}

Termin: 15. Juni 2009

Ort: Ort wird noch bekanntgegeben

Teilnahme kostenfrei

\section{Kanalinspektionskurs gemäß} ÖNORM EN 13508-2

Termin: 22.-26. Juni 2009

Ort: Wien

Kosten (+ $20 \%$ USt.): ÖWAV-Mitglieder Euro 750,--, Nichtmitglieder Euro 950,-

\section{Auffrischungskurs für Klärwärter}

Termin: 15.-17. Juli 2009

Ort: Wien

Kosten (+ $20 \%$ USt.): ÖWAV-Mitglieder Euro 480,-, Nichtmitglieder Euro 680,-

\section{Klärwärter-Fortbildungskurs}

Termin: 31. August-4. September 2009

Ort: Wien

Kosten (+ $20 \%$ USt.): ÖWAV-Mitglieder Euro 650,--, Nichtmitglieder Euro 850,- 
2. ÖWAV-Kurs „Anwendung von ÖWAVRegelblatt 19 (neu) - Richtlinien zur Bemessung von Mischwasserentlastungen in der Praxis"

Termin: 28.-29. September 2009

Ort: Wien

\section{Laborpraktikum für Klärwärter}

Termin: 28. September-2. Oktober 2009

Ort: Linz-Asten

Kosten (+ 20 \% USt.): ÖWAV-Mitglieder Euro 910,-, Nichtmitglieder Euro 1.060,-

\section{Maschinentechnischer Kurs für} Klärwärter

Termin: 5. -9. Oktober 2009

Ort: Linz-Asten

Kosten (+ $20 \%$ USt.): ÖWAV-Mitglieder Euro 520,-, Nichtmitglieder Euro 670,-

\section{VÖEB-ÖWAV-Kanaldichtheitsprüfungskurs}

Termin: 12.-14. Oktober 2009

Ort: Anif

Kosten (+ $20 \%$ USt.): ÖWAV-/VÖEB-Mitglieder Euro 620,-, Nichtmitglieder Euro 750,-

Informationen und Anmeldung:

VÖEB, Tel. +43-1-713 02 53, Fax +43-1-715 21 07, voeb@voeb.at, www.voeb.at

\section{ABFALL}

\section{Praxisworkshop für Betreiber und} Betriebspersonal von Kompostierungsanlagen: Qualitätsmanagement Dokumentation - Aufzeichnung

Termin: 7.-8. Mai 2009

Ort: Linz

Kosten (+ $20 \%$ USt.): ÖWAV-Mitglieder Euro 390,-, Nichtmitglieder Euro 540,-
3. Ergänzungskurs zur Deponieverordnung 2008 für Absolventen des Ausbildungskurses „Baurestmassen - Ausbildung von Deponiepersonal“"

Termin: 26. Mai 2009

Ort: Wien

Kosten (+ $20 \%$ USt.): ÖWAV-/BRV-Mitglieder Euro 260,-, Nichtmitglieder Euro 460,-

\section{3. ÖWAV-Ausbildungskurs „Deponie-Ein- gangskontrolle für das Betriebspersonal von Massenabfall- und Reststoffdeponien"}

Termin: 22.-26. Juni 2009

Ort: Linz-Asten

Kosten (+ 20 \% USt.): ÖWAV-Mitglieder Euro 685,--, Nichtmitglieder Euro 870,-

4. Ergänzungskurs zur Deponieverordnung 2008 für Absolventen des Ausbildungskurses „Baurestmassen - Ausbildung von Deponiepersonal“"

Termin: 30. Juni 2009

Ort: Wien

Kosten (+ $20 \%$ USt.): ÖWAV-/BRV-Mitglieder Euro 260,-, Nichtmitglieder Euro 460,-

TALSPERREN

7. Kurs „Sicherheit von kleinen Stau- und Sperrenanlagen - Kurs für Verantwortliche im Bereich Bau, Betrieb und Überwachung"

Termin: 6. -7. Mai 2009

Ort: Sillian (Osttirol)

Kosten (+ $20 \%$ USt.): ÖWAV-/ATCOLD-Mitglieder Euro 550,-, Nichtmitglieder Euro 750,-

Weitere Informationen und Anmeldung:

Gesellschaft für Wasser- und Abfallwirtschaft GmbH des ÖWAV, 1010 Wien, Marc-Aurel-Straße 5, Isabella Seebacher. Tel. 01/535 57 20-82, seebacher@ oewav at oder www. oewav at $>>$ Veranstaltungen

Aufgrund der Voranmeldung erhalten Sie das detaillierte Veranstaltungsprogramm. 